Published in final edited form as:

Anal Chem. 2017 February 07; 89(3): 1399-1404. doi:10.1021/acs.analchem.6b04337.

\title{
Visualization, Quantification, and Alignment of Spectral Drift in Population Scale Untargeted Metabolomics Data
}

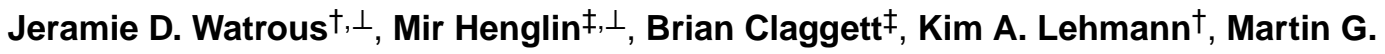 \\ Larson $\S, \|$, Susan Cheng $\$, \S,{ }^{\ddagger}, \perp$, and Mohit Jain ${ }^{\dagger,},{ }^{*}, \perp$ \\ tDepartments of Medicine and Pharmacology, University of California San Diego, La Jolla, \\ California 92093, United States \\ ¥Cardiovascular Division, Department of Medicine, Brigham and Women’s Hospital, Harvard \\ Medical School, Boston, Massachusetts 02115, United States \\ §Framingham Heart Study, Framingham, Massachusetts 01702, United States \\ "Biostatistics Department, School of Public Health, Boston University, Boston, Massachusetts \\ 02118, United States
}

\begin{abstract}
Untargeted liquid-chromatography-mass spectrometry (LC-MS)-based metabolomics analysis of human biospecimens has become among the most promising strategies for probing the underpinnings of human health and disease. Analysis of spectral data across population scale cohorts, however, is precluded by day-to-day nonlinear signal drifts in LC retention time or batch effects that complicate comparison of thousands of untargeted peaks. To date, there exists no efficient means of visualization and quantitative assessment of signal drift, correction of drift when present, and automated filtering of unstable spectral features, particularly across thousands of data files in population scale experiments. Herein, we report the development of a set of R-based scripts that allow for pre- and postprocessing of raw LC-MS data. These methods can be integrated with existing data analysis workflows by providing initial preprocessing bulk nonlinear retention time correction at the raw data level. Further, this approach provides postprocessing visualization and quantification of peak alignment accuracy, as well as peak-reliability-based parsing of processed data through hierarchical clustering of signal profiles. In a metabolomics data set derived from $~ 3000$ human plasma samples, we find that application of our alignment tools resulted in substantial improvement in peak alignment accuracy, automated data filtering, and
\end{abstract}

\footnotetext{
*Corresponding Authors: scheng@ rics.bwh.harvard.edu. mjain@ucsd.edu.

Author Contributions

J.D.W. and M.H. and S.C. and M.J. contributed equally.

ORCID

Jeramie D. Watrous: 0000-0001-9124-6783

The authors declare no competing financial interest.

This set of annotated R-based scripts is available for the metabolomics community at http://www.jainlaboratory.org/tools/ alignmentscripts.html, along with user documentation and details.

Supporting Information

The Supporting Information is available free of charge on the ACS Publications website at DOI: 10.1021/acs.analchem.6b04337. Experimental methods, list of isotopically labeled internal standards, endogenous landmark peak examples, XCMS and Mzmine output comparison for corrected versus uncorrected data, heatmaps from statistical outcomes analysis, and example output of feature clustering (PDF)
} 
ultimately statistical power for detection of metabolite correlates of clinical measures. These tools will enable metabolomics studies of population scale cohorts.

\section{Graphical Abstract}



Metabolomics is among the most promising strategies for deep phenotyping of complex biological samples. ${ }^{1-3}$ When applied to human biospecimens, particularly across population scale cohorts of thousands of individuals, metabolomics allows for identification of biomarkers years prior to onset of disease, understanding of disease pathophysiology, elucidation of mechanisms of action for pharmacologic agents, and even a means for personalization of therapy. ${ }^{1-11}$ Continual advances in high resolution liquid-

chromatography-mass spectrometry (LC-MS) enable routine separation and measurement of thousands of discrete chemical features from biospecimens in an "untargeted" fashion without the need for chemical labeling, enabling broad and comprehensive coverage of the metabolome. ${ }^{12-15}$ As is the case with any analytical approach, even the most well controlled LC-MS methods will experience fluctuations ("signal drift") during constant measurement of thousands of unique samples over extended periods of continuous analysis time. ${ }^{16-20}$ It is therefore essential that major sources of drift, including changes in instrument sensitivity, chromatographic retention time, and sample extraction efficiency, be accounted for during analysis to allow for comparison of individual spectral peaks across all samples, to limit errors in signal processing, and to avoid introduction of systematic artifacts. ${ }^{18,21}$ The major source of signal drift over an extended LC-MS run arises from nonlinear fluctuations in LC retention time, resulting from column aging, slight variability in mobile phase preparations, and differences in sample matrix complexity, all of which can influence a given metabolites interaction with the LC column's stationary phase. ${ }^{19,22-25}$ Similarly, with untargeted metabolomics, a certain subpopulation of metabolites will inevitably be chemically unstable under any given method parameters, resulting in rapid time-dependent changes in signal intensity $16,20,21$ as well as irregularity in sample recovery during preparation (i.e., batch effects). ${ }^{17,26}$ Together, these signal drifts are amplified in population scale untargeted metabolomics studies across thousands of samples. Accurate extraction and interpretation of population scale LC-MS data requires robust, automated methods for detection, visualization, and quantification of signal drift.

Given the ubiquitous nature of these limitations in LC-MS metabolomics experiments, significant effort has been put forth in creating software for handling signal normalization 
due to instrument drift ${ }^{26-29}$ and batch effects ${ }^{17,30-32}$ as well as creating QC metrics for evaluating LC-MS stability and reliability. ${ }^{18,20,21,33}$ Similarly, prior efforts have also reported approaches for addressing nonlinear retention time correction. ${ }^{19,22-24}$ While most of these tools provide valuable capabilities in data processing, we often find they are not able to be easily incorporated with established analysis pipelines. More importantly, existing methods are generally not capable of processing thousands of LC-MS files in a single analysis, a requirement for population scale metabolomic studies. In addition, many reported approaches also require the use of vendor specific data formats or abstract file conversions which severely limit options for further downstream data processing. To combine data handling tools into an integrated software pipeline, open source analysis suites such as XCMS,${ }^{25}$ Mzmine, ${ }^{34}$ OpenMS, ${ }^{35}$ and SMART ${ }^{36}$ have been developed to allow users the ability to perform nonlinear peak alignment with downstream tools for peak picking and normalization. However, a common shortcoming of many of these packages is their inability to perform computationally intensive functions (e.g., as unsupervised nonlinear retention time correction) on thousands of data files in a single analysis as well as advanced data quality filtering such as the ability to determine signal reliability based on a peaks cumulative signal profile.

To expand functionality and flexibility of data handling across thousands of untargeted LCMS files, we sought to create a suite of simple software modules that could be easily added to and incorporated with existing analysis pipelines. The first module allows for initial preprocessing and bulk nonlinear retention time correction of raw spectral data using user indicated landmark peaks. Corrected data can thereafter be imported into any user preferred compatible software improving the quality of secondary unsupervised nonlinear alignment as well as allowing for the use of less computationally intensive linear peak alignment algorithms. The second module evaluates the quality of peak alignment, allowing rapid setting optimization and assessment of data quality. Lastly, the third module allows for filtering of data based on signal reliability by performing hierarchical clustering on peak profile patterns and sorting based on the type of drift (e.g., batch effects, chemical/ thermal instability, and misalignment). This allows for each subset of data to be normalized using available software specifically designed for correcting that type of drift. These R-based scripts were tested and validated using a population scale data set comprised of 2895 human plasma samples, with substantial improvement in peak alignment accuracy and automated data filtering, ultimately resulting in greater statistical power for identifying correlates between observed metabolites and clinical measures. These modules are provided to the metabolomics community to enable population-based metabolomics studies.

\section{RESULTS AND DISCUSSION}

\section{Prealignment of LC-MS Data}

Analysis of LC-MS data is typically performed by detecting all masses within each scan, extracting each mass individually and determining if chromatographic peaks are present within the mass trace (i.e., peak picking), aligning corresponding peaks between samples (i.e., feature alignment), removing unreliable chromatographic features (typically through basic filtering as well as manual inspection), and performing statistical analysis with respect 
to phenotypes (Figure 1A). ${ }^{37}$ While mass detection and peak picking algorithms are relatively robust and consistent across most software suites, feature alignment and signal filtering can differ greatly in terms of approach and capability. Accurate pairing of corresponding chromatographic features among not only sequentially analyzed samples but also subsequent data sets requires highly accurate modeling of complex nonlinear retention time drift. ${ }^{19,22-24}$ Failure to account for this drift may introduce systematic errors in signal processing such as spectral features becoming "split" where their retention times have drifted outside of the allowable tolerance window causing the software to treat one feature as multiple features (Figure 1B). Similarly, when neighboring isobaric peaks are present, different features may become cross-aligned whereby signal from different compounds is treated as a single feature (Figure 1B). Moreover, we have observed that most nonlinear unsupervised alignment algorithms do not offer sufficient flexibility in the alignment model to adjust for minor errors in final alignment. We therefore sought to create a supervised, landmark-based, nonlinear, preprocessing alignment algorithm that allows for bulk retention time correction in order to improve the accuracy of secondary alignment. To examine the extent of retention drift present in "real world" metabolomics data, LC-MS data was collected from 2895 human plasma samples, prepared, and analyzed as 33 batches of 93 samples each. Absolute retention time drift for 24 isotopically labeled internal standards (Table S-1) acting as known landmarks and 60 endogenous unknown landmark features (i.e., ubiquitous peaks present in every sample with little variability) were plotted against run retention time for each batch to reveal nonlinear drift patterns with variability in these patterns among prepared batches (Figures 2A and S-1). Given the complexity and size of data, low order polynomials do not offer sufficient degrees of freedom to accurately account for drift across the entire run time and changes in this drift profile between samples necessitate that each sample be corrected independently. We therefore employed running average cubic smoothing splines as the foundation for building drift models with varying degrees of freedom (i.e., 4, 8, 16, and 32 degrees of freedom). Resulting models using either internal standard landmarks or endogenous landmarks were highly comparable (data not shown), and therefore, all landmarks were used for analysis. Using these models, the scan retention times within each .mzXML raw data file were modified to fit each model and were exported as new corrected .mzXML files, which could then be imported into any compatible software suite. To test the accuracy and quality of alignment, a metric was established for quantification of feature misalignment. Major shifts in retention time typically occur between batches, and as such, we searched for mass features that were statistically absent from within at least one of the 33 batches. These batch-specific perturbations were visualized by plotting the percent misalignment versus cumulative metabolite number and quantified by calculating the area under the curve (AUC) for each trace (Figure 2B). Prealigned ("corrected") and uncorrected, raw data were loaded in Mzmine 2.21, and all spectra features above 50000 counts (roughly 6000 peaks) were aligned using the Join Aligner linear alignment function. ${ }^{34}$ Quantitation of misalignment revealed that $82 \%$ of peaks were misaligned within the uncorrected data set, confirming the ubiquitous nature of alignment issues in untargeted metabolomics data. In contrast, only $14 \%$ were misaligned in the corrected data using 16-degrees of freedom. Figure $2 \mathrm{C}$ shows example extracted ion chromatograms for $\mathrm{m} / \mathrm{z} 269.1782$, a mass that exhibits an abundance of isobaric species, from each of the 33 batches, both before and after retention time correction is applied, with 
marked improvement following institution of the correction model. We further examined whether landmark-based prealignment improves more sophisticated, nonlinear alignment tools that are present in common processing platforms such as Mzmine (RANSAC algorithm ${ }^{34}$ ) or XCMS online (https://xcmsonline.scripps.edu). ${ }^{25}$ Analysis of the entire metabolomics data set of $\sim 3000$ files was not possible, however, given the size and memory constraints present with both of these processing platforms. A subset of 200 random metabolomics data files was therefore selected and examined. Metabolomics data corrected using our landmark alignment preprocessing step improved data quality and overall alignment, with fewer missing values with both XCMS (Figure S-2) and Mzmine RANSAC (Figure S-3). One of the major advantages of performing retention time correction within the raw data before peak deconvolution is that the end user is able to import the corrected data into their desired data analysis platform, regardless of the strength of its alignment algorithms. As such, prealignment of population scale metabolomics data using a facile drift model may greatly improve data quality and reduce systematic error due to improper signal processing.

\section{Statistical Association of Aligned Metabolomics Data}

Typically, analysis software equipped with simple linear or low order polynomial-based alignment algorithms requires retention time limits to be set using a large enough time window to encompass all possible drift within the raw data. This can drastically increase the risk of cross-alignment with neighboring isobaric features. For population scale metabolomics analysis, this cross alignment can have major implications when correlating spectral information with clinical outcomes and can lead to both false positives and false negatives, undermining the overall analysis. To determine the extent to which the optimal alignment algorithm impacts the results of metabolite associations assessed in a large population scale cohort, corrected and uncorrected LC-MS data for 2895 patient plasma samples were loaded into Mzmine 2.21 and features were aligned using a simple linear alignment algorithm with a 0.025 min window for corrected data and a 0.15 min window for the uncorrected data (as 0.15 min represents the maximum observed retention time drift in the raw data for the 2895 samples). We then performed linear and logistic regression analyses to examine the associations of each metabolite with the common clinical features of age and sex, respectively. Corrected raw data showed marked improvement in association with clinical phenotype, especially with respect to the magnitude of associations assessed by beta coefficients (Figure S-4A) and statistical significance of associations indicated by $P$ values (Figure S-4B). For detecting metabolites associated with age, a continuous outcome, a total of $13.8 \%$ of all metabolites measured reached the Bonferroni threshold of statistical significance when models were run using the uncorrected data and $18.0 \%$ when models were run using corrected data $(P=0.002)$. When compared to the results of models run using corrected data, the models run using uncorrected data produced $8.9 \%$ false negative results and $27.5 \%$ false positive results. Similar degrees of high false positive and false negative levels were observed with uncorrected metabolite association with gender, a binary outcome (data not shown). We therefore find that proper prealignment of untargeted metabolomics data substantially improves both sensitivity and specificity for detecting potentially important metabolite associations in population scale metabolomics analyses. 


\section{Visualization and Hierarchical Clustering of Aligned Spectra Features}

Even when properly integrated and aligned, signal drift may be introduced into untargeted metabolomics data sets due to nonprocessing errors, including day-to-day variances in sample preparation (batch effects), changes in instrument sensitivity, or even simple chemical instability and breakdown of unstable metabolites during the time between extraction and LC-MS analysis. ${ }^{16-18,21,33}$ While methods are available for unsupervised correction of signal drifts, ${ }^{16,17,22,24}$ to the best of our knowledge, there does not exist a facile method for parsing out LC-MS signal profiles based on the type of drift they exhibit so that further processing can be performed on the basis of the type of drift or removed if the drift is not correctable. We reasoned that metabolites subject to batch effects, instrument drift, or chemical instability may demonstrate predictable patterns across large scale metabolomics experiments (Figure 3A) and that metabolites exhibiting similar patterns may be visualized and clustered to potentially identify and remove unreliable signals from further analysis. Using data from the 2895 human plasma samples following nonlinear retention time correction, the mean signal intensity from the resulting $\sim 6000$ spectral features was plotted with respect to run order. Trace patterns across run order were clustered, and those metabolites that demonstrated significant fluctuations within a batch or across batches were automatically grouped together. Resulting metabolite clusters were then parsed into groups based on signal reliability with reliable signals colored blue and signals exhibiting significant levels of deviation within their intensity profiles colored red and labeled as unreliable, allowing for rapid visualization of untargeted metabolomics data (Figure S-5). Figure 3B highlights several examples of such signals with profiles for stable metabolites being relatively invariant from batch-to-batch cross aligned metabolites, whereas misaligned peaks exhibited batch "drop out"; those metabolites sensitive to batch to batch variance in sample preparation exhibited clear interbatch shifts, and unstable metabolites exhibited both intra- and interbatch decrement in signal. This approach not only allows for global visualization of data quality within an experiment but also enables unbiased automatic parsing of features into groups based on their signal "reliability".

\section{CONCLUSIONS}

Proper feature alignment and peak quality filtering in LC-MS data sets is critically important for correctly analyzing and interpreting spectral information, particularly across untargeted metabolomics data sets including those used for chemotyping of population scale clinical cohorts. We have shown that performing bulk preprocessing retention time correction at the raw data level not only allows end users freedom to utilize analysis software lacking robust alignment algorithms but also provides better quality data extraction when used prior to secondary processing with software suites such as XCMS or Mzmine. In addition, the ability to visualize and quantitate the degree of misalignment within a data set before further processing is a critical checkpoint within any analysis pipeline. Lastly, using hierarchical clustering to categorize signals based on the shape of the signal intensity profile across batches allows for visualization and parsing of data based on signal reliability. These approaches can enhance the quality of LC-MS data analysis with improved chromatographic alignment, thereby allowing for increased accuracy in feature quantification, automated parsing of unreliable features, and greater downstream statistical power. 


\section{Supplementary Material}

Refer to Web version on PubMed Central for supplementary material.

\section{Acknowledgments}

This work was supported by funding from the National Institutes of Health (R01 HL134168: S.C., M.J.), the American Heart Association (CVGPS Pathway Award: S.C., M.J.), the Tobacco-Related Disease Research Program (\#24RT-0032: M.J.; 24FT-0010: J.D.W.), the American Cancer Society (IRG Grant \#70-002: M.J.), and a Doris Duke Charitable Foundation Grant (\#2015092: M.J., S.C.).

\section{References}

1. Johnson CH, Ivanisevic J, Siuzdak G. Nat Rev Mol Cell Biol. 2016; 17:451-459. [PubMed: 26979502]

2. Nicholson JK, Holmes E, Kinross JM, Darzi AW, Takats Z, Lindon JC. Nature. 2012; 491:384-392. [PubMed: 23151581]

3. Nagana Gowda GA, Raftery D. Curr Metabolomics. 2013; 1:227-240. [PubMed: 27134822]

4. Cheng S, Larson MG, McCabe EL, Murabito JM, Rhee EP, Ho JE, Jacques PF, Ghorbani A, Magnusson M, Souza AL, Deik AA, Pierce KA, Bullock K, O’Donnell CJ, Melander O, Clish CB, Vasan RS, Gerszten RE, Wang TJ. Nat Commun. 2015; 6:6791-6799. [PubMed: 25864806]

5. Wang TJ, Larson MG, Vasan RS, Cheng S, Rhee EP, McCabe E, Lewis GD, Fox CS, Jacques PF, Fernandez C, O’Donnell CJ, Carr SA, Mootha VK, Florez JC, Souza A, Melander O, Clish CB, Gerszten RE. Nat Med. 2011; 17:448-453. [PubMed: 21423183]

6. Kaddurah-Daouk R, Kristal BS, Weinshilboum RM. Annu Rev Pharmacol Toxicol. 2008; 48:653683. [PubMed: 18184107]

7. Kaddurah-Daouk R, Weinshilboum RM. Clin Pharmacol Ther. 2014; 95:154-167. [PubMed: 24193171]

8. Snowden S, Dahlen SE, Wheelock CE. Bioanalysis. 2012; 4:2265-2290. [PubMed: 23046268]

9. Suhre K, Shin SY, Petersen AK, Mohney RP, Meredith D, Wagele B, Altmaier E, Deloukas P, Erdmann J, Grundberg E, Hammond CJ, de Angelis MH, Kastenmuller G, Kottgen A, Kronenberg F, Mangino M, Meisinger C, Meitinger T, Mewes HW, Milburn MV, Prehn C, Raffler J, Ried JS, Romisch-Margl W, Samani NJ, Small KS, Wichmann HE, Zhai G, Illig T, Spector TD, Adamski J, Soranzo N, Gieger C. Nature. 2011; 477:54-60. [PubMed: 21886157]

10. Shah SH, Kraus WE, Newgard CB. Circulation. 2012; 126:1110-1120. [PubMed: 22927473]

11. Beger RD, Dunn W, Schmidt MA, Gross SS, Kirwan JA, Cascante M, Brennan L, Wishart DS, Oresic M, Hankemeier T, Broadhurst DI, Lane AN, Suhre K, Kastenmüller G, Sumner SJ, Thiele I, Fiehn O, Kaddurah-Daouk R. Metabolomics. 2016; 12:149. [PubMed: 27642271]

12. Alonso A, Marsal S, Julià A. Front Bioeng Biotechnol. 2015; 3:23. [PubMed: 25798438]

13. Zamboni N, Saghatelian A, Patti GJ. Mol Cell. 2015; 58:699-706. [PubMed: 26000853]

14. Patti GJ, Yanes O, Siuzdak G. Nat Rev Mol Cell Biol. 2012; 13:263-269. [PubMed: 22436749]

15. Johnson CH, Ivanisevic J, Benton HP, Siuzdak G. Anal Chem. 2015; 87:147-156. [PubMed: 25389922]

16. Fernandez-Albert F, Llorach R, Garcia-Aloy M, Ziyatdinov A, Andres-Lacueva C, Perera A. Bioinformatics. 2014; 30:2899-2905. [PubMed: 24990606]

17. Wang SY, Kuo CH, Tseng YJ. Anal Chem. 2013; 85:1037-1046. [PubMed: 23240878]

18. Gika HG, Theodoridis GA, Wingate JE, Wilson ID. J Proteome Res. 2007; 6:3291-3303. [PubMed: 17625818]

19. Podwojski K, Fritsch A, Chamrad DC, Paul W, Sitek B, Stuhler K, Mutzel P, Stephan C, Meyer HE, Urfer W, Ickstadt K, Rahnenfuhrer J. Bioinformatics. 2009; 25:758-764. [PubMed: 19176558]

20. Fang M, Ivanisevic J, Benton HP, Johnson CH, Patti GJ, Hoang LT, Uritboonthai W, Kurczy ME, Siuzdak G. Anal Chem. 2015; 87:10935-10941. [PubMed: 26434689] 
21. Gika HG, Theodoridis GA, Earll M, Snyder RW, Sumner SJ, Wilson ID. Anal Chem. 2010; 82:8226-8234. [PubMed: 20828141]

22. Kirchner M, Saussen B, Steen H, Steen JAJ, Hamprecht FA. J Stat Soft. 2007; 18:1.

23. Lange E, Tautenhahn R, Neumann S, Gröpl C. BMC Bioinf. 2008; 9:375.

24. Nordström A, O’Maille G, Qin C, Siuzdak G. Anal Chem. 2006; 78:3289-3295. [PubMed: 16689529]

25. Smith CA, Want EJ, O’Maille G, Abagyan R, Siuzdak G. Anal Chem. 2006; 78:779-787. [PubMed: 16448051]

26. Ejigu BA, Valkenborg D, Baggerman G, Vanaerschot M, Witters E, Dujardin JC, Burzykowski T, Berg M. OMICS. 2013; 17:473-485. [PubMed: 23808607]

27. Karpievitch YV, Nikolic SB, Wilson R, Sharman JE, Edwards LM. PLoS One. 2014; 9:e116221. [PubMed: 25549083]

28. Veselkov KA, Vingara LK, Masson P, Robinette SL, Want E, Li JV, Barton RH, Boursier-Neyret C, Walther B, Ebbels TM, Pelczer In, Holmes E, Lindon JC, Nicholson JK. Anal Chem. 2011; 83:5864-5872. [PubMed: 21526840]

29. Sysi-Aho M, Katajamaa M, Yetukuri L, Orešič M. BMC Bioinf. 2007; 8:93.

30. Kuligowski J, Pérez-Guaita D, Lliso I, Escobar J, León Z, Gombau L, Solberg R, Saugstad OD, Vento M, Quintás G. Talanta. 2014; 130:442-448. [PubMed: 25159433]

31. Kuligowski J, Sánchez-Illana Á, Sanjuán-Herráez D, Vento M, Quintás G. Analyst. 2015; 140:7810-7817. [PubMed: 26462549]

32. Wehrens R, Hageman JA, van Eeuwijk F, Kooke R, Flood PJ, Wijnker E, Keurentjes JJB, Lommen A, van Eekelen HDLM, Hall RD, Mumm R, de Vos RCH. Metabolomics. 2016; 12:88. [PubMed: 27073351]

33. Fang ZZ, Gonzalez FJ. Arch Toxicol. 2014; 88:1491-1502. [PubMed: 24710571]

34. Pluskal T, Castillo S, Villar-Briones A, Oresic M. BMC Bioinf. 2010; 11:395.

35. Sturm M, Bertsch A, Gröpl C, Hildebrandt A, Hussong R, Lange E, Pfeifer N, Schulz-Trieglaff O, Zerck A, Reinert K, Kohlbacher O. BMC Bioinf. 2008; 9:163.

36. Liang YJ, Lin YT, Chen CW, Lin CW, Chao KM, Pan WH, Yang HC. Anal Chem. 2016; 88:63346341. [PubMed: 27248514]

37. Zhou B, Xiao JF, Tuli L, Ressom HW. Mol BioSyst. 2012; 8:470-481. [PubMed: 22041788] 
A



B

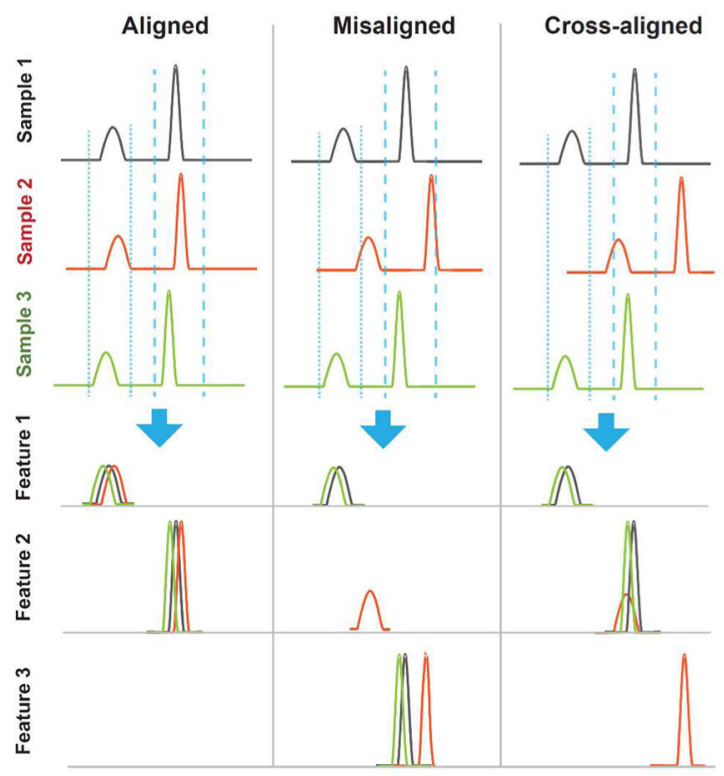

Figure 1.

Misalignment of spectral data. (A) Typical LC-MS data analysis workflow. Steps encompassed in the red box indicated steps where drift correction was applied. (B) Possible outcomes of improper alignment. Left column shows proper alignment of the two peaks within the retention time window (dashed lines), whereas misaligned (center) and crossaligned (right) peaks are shown along with their impact on final data outcomes. 
A



C



B



Linear Alignment of Corrected Data

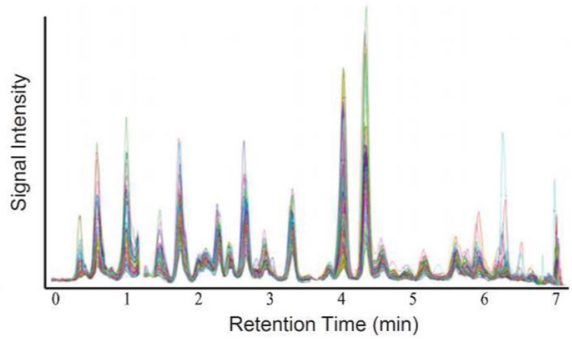

Figure 2.

Quantification of signal drift. (A) Modeling nonlinear retention time drift across four representative sample preparation batches $(N \sim 90$ samples per batch). The blue line indicates mean retention drift relative to a reference sample whereas the clusters of black points indicate individual retention times for the 24 internal standards and 60 landmark peaks. Drift models were generated for each of the 2895 samples and used to create corrected .mzXML files. (B) Quantifying peak alignment quality by plotting the percent of misaligned features based on batch-to-batch consistency within randomized patient samples and calculating the area under the curve (AUC) with a higher AUC value indicating better alignment. (C) Example overlay of EIC's from each of the 33 batches for $m / z 269.1782$, which displays many isobaric structures, before and after retention time correction. 
A
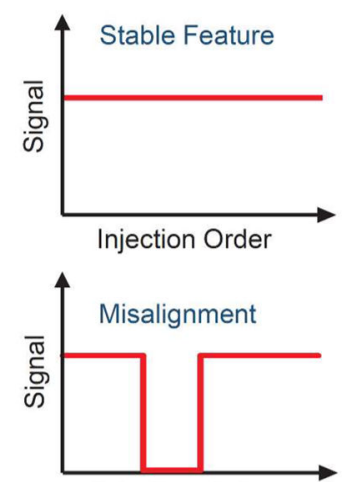

Injection Order

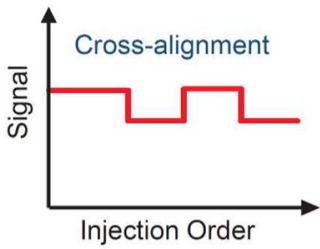

B

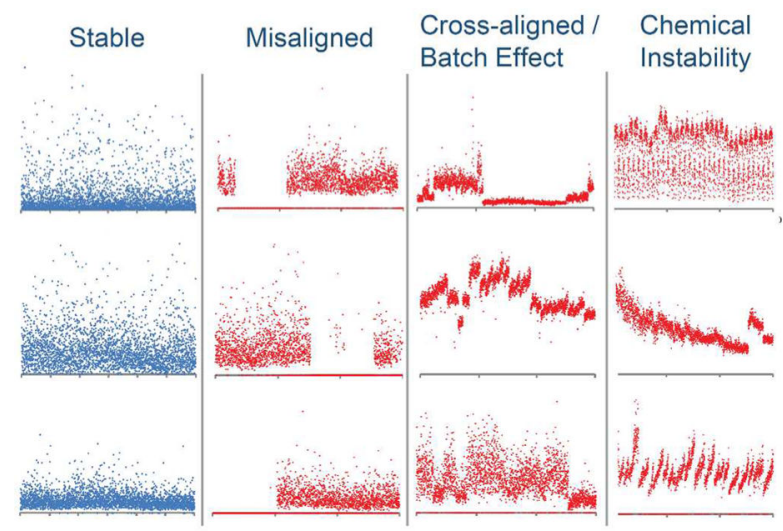

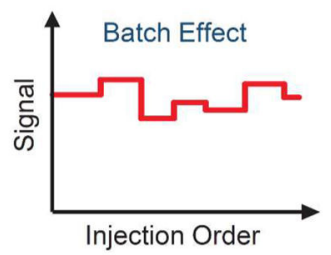

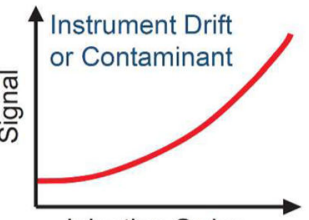

Injection Order

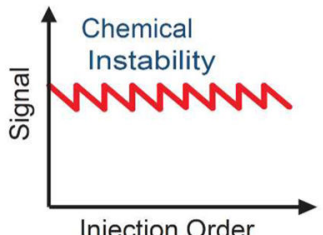

Injection Order

Figure 3.

Hierarchical clustering-based filtering of unreliable signals. (A) Examples of possible intensity patterns that can occur during a large scale LC-MS experiment. Red line indicates mean intensity value for a chromatographic feature across all batches. (B) Examples of parsed clustering output showing stable features (consistent means across all batches), misaligned features (signal loss across one or more batches), cross-aligned and batch affected features (regular or erratic shifting of mean signal between batches), and chemically unstable features (gradual increase or decrease in signal over each batch or across the entire experiment). 